\title{
Development of Reliability Indices for Electric Distribution Network in Egypt
}

\author{
Eman Ahmed, Sherein Abdualla, Kamelia Youssef and Hatem Waheed \\ Egyptian Electric Utility and Consumer Protection Regulatory Agency \\ sharino@hotmail.com, white_storm100@yahoo.com
}

\begin{abstract}
Reliability indices (RIs) are the elemental benchmark used by Egyptian Electricity Holding Company (EEHC), and the Electric Utility and Consumer Protection Regulatory Agency (Egypt ERA) to evaluate the continuity and compliance of supply, which surpasses the customer's requirements and satisfaction.
\end{abstract}

The power system is very complex, mixing huge different types of generating resources and clusters to supply electric power through transmission and distribution system to a number of customers with varying requirements.

The main function of electric system is to supply customers with electric energy that has an acceptable degree of reliability and quality. The power system continuity of supply level is controlled through system indices. The most widely used reliability indices are SAIFI, SAIDI and CAIDI (IEEE std. 1366-2000).

Historical electrical indices, reliability indices threshold and satisfaction index are used as guide for electric network performance, which measure the adequate and secure power supply.

The paper presents the reliability indices, relation between indices and satisfaction area to highlight the appropriate guideline values for electric systems, also presents the development of indices since 2011 to now for distribution network in Egypt.

Keywords - Reliability; Power System; Benchmark; Continuity; Indicators.

\section{INTRODUCTION}

The Egyptian Electricity Holding Company (EEHC) mission towards the society is to supply electricity to all types of consumers according to international performance standards taking into consideration all environmental, social and economic determinates and also the terms and conditions set by The Egyptian Electric Utility and Consumer Protection Regulatory Agency (EGYPTERA).

EEHC has sixteen affiliated companies as shown in "Fig. 1" (six generations (EPC), nine distributions and the Egyptian Electricity Transmission Company $(\mathrm{ETC}))$.

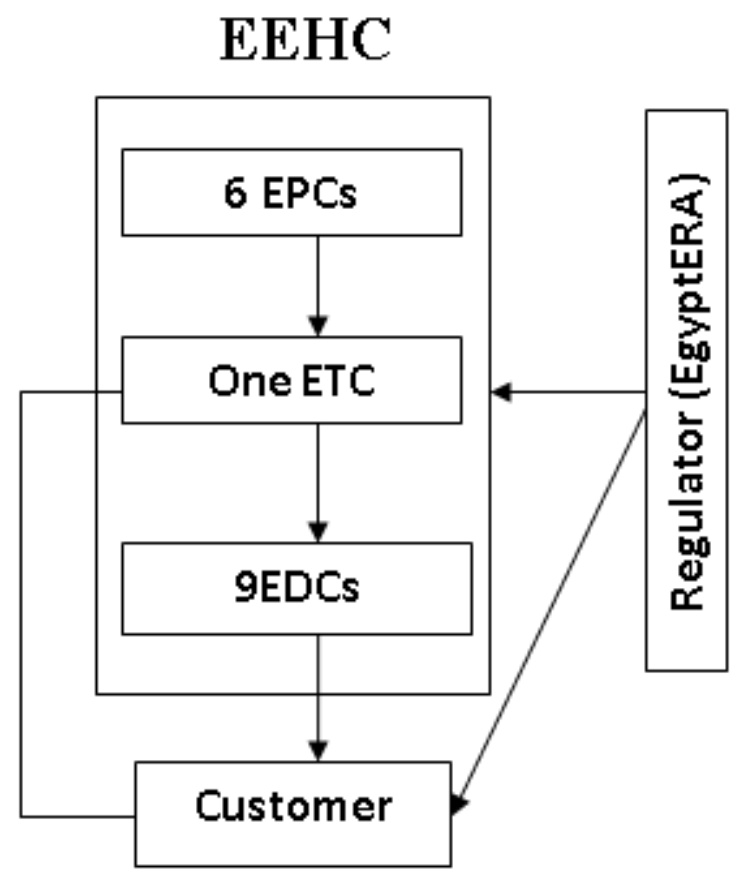

Fig. 1. the relation between EEHC and Egypt ERA

The main objectives of electricity Distribution Company

$(E D C)$ are:

- Distributing and selling to consumers on medium and low voltages.

- Managing, operating and maintaining medium and low voltages grids in the company.

- Ensuring high level of quality, availability and continuity of supply to distribution customers. 
- The following procedures have been taken to improve

- Continuity of supply indices:

- Analysis of the causes in case of increased unplanned interruptions and relate it to network renovation and Rehabilitation plans.

- Follow up the implementation of maintenance programs to insure optimizations of interruption of supply time and at the same time implementation of the maintenance procedures with high quality.

- Intensive field inspections and data collection for interruptions.

Each EDC consists of number of geographical sectors, Have number of districts. The district network consists of MV distributors, distribution transformers (MV/LV), and MV, LV lines, as shown in "Fig.2".

EEHC and EGYPTERA use Rls to trace the Performance of EDCs. EGYPTERA most commonly uses KPIs: SAIFI, SAIDI, CAIDI, ENS and CENS.

\section{PERFORMANCE INDICES (PIS)}

Recommended interruption indices or key performance indices (PIs) are those defined in both IEEE 1366[1] and the CIGRE study committee C2 [2]:

- $\quad$ SAIFI (System Average Interruption Frequency Index): the average number of sustained interruptions per customer during the year.

- SAIDI (System Average Interruption Duration Index): the average time for which customers power supply is interrupted in a year.

- $\quad$ CAIDI (Customer Average Interruption Duration Index): the average time required to restore service to the average customer per sustained interruption.

- $\quad$ ENS (Energy Not Supplied): the summation of energy not supplied due to supply interruptions over a year Period. Its cost is CENS (cost of energy not supplied).

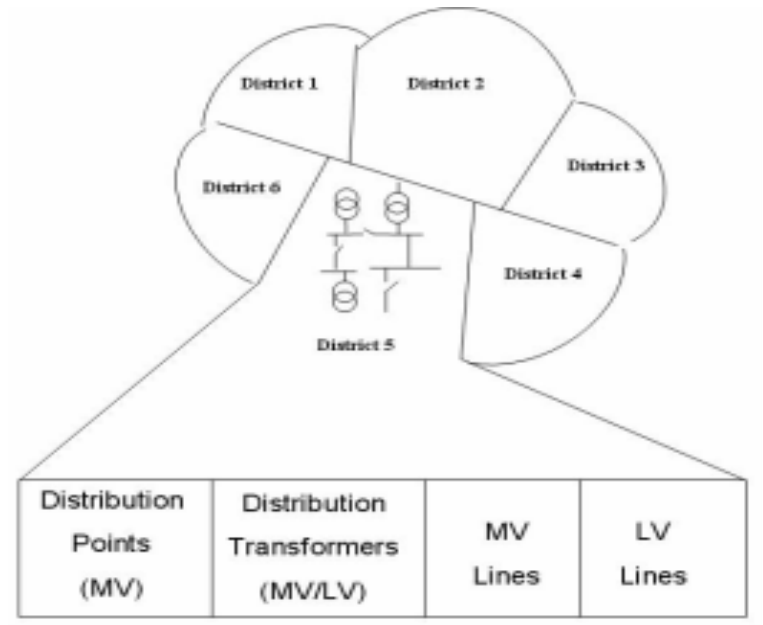

Fig. 2. Part of geographical area of an EDC

\section{SYSTEM RELIABILITY INDICES (RIS)}

\section{A. System Average Interruption Frequency Index} (SAIFI)

It measures the average number of interruptions experienced by each customer. All planned and unplanned interruptions are used in calculating the index. SAIFI can be calculated as follows:

SAIFI $=$ (No. of interruptions during one year)/ (No. of customers).

\section{B. System Average Interruption Duration Index (SAIDI)}

It measures the yearly average interruptions duration per customer. It can be calculated as follows:

SAIDI $=\left(\sum\right.$ duration of interruption in $\left.\mathrm{min}\right) /$ (No. of customers).

\section{Customer Average Interruption Duration Index (CAIDI)}

It measures the average time required to restore service to the average customer per interruption. The following formula is employed for calculating CAIDI: CAIDI $=($ SAIDI $) /($ SAIFI). [4], [5]

\section{CONTINUITY OF POWER SUPPLY}

The main aspects of quality for electric network operation are continuity of supply, safety, technical quality of the commodity, end user service and environmental impact. Continuity of supply measures the electric networks ability to supply the end users with electricity. It is generally characterized as the frequency and duration of interruptions in supply. 
- $\quad$ Continuity of power supply or sustained outage an outage that lasts longer than a specified amount of time .SAIDI, SAIFI and CAIDI are all based on sustained outage .The duration of a sustained outage varies from state to state. IEEE -1366 defines the duration of sustained outage to be 5 minutes.

- Power quality - measure of the purity of the electric waveform on power lines. A power quality event, which is not the same as an outage, occurs when one of the waveforms differs from a pure sinusoidal waveform or one or two phases of power are lost. [10]

Measurements that can quantify power quality are harmonic distortion and peak to peak voltage. Power quality events can last from few cycles to a few seconds and can be caused by lightning strikes, falling trees, utility operations and operations from other customers such as disturbances from starting a large motor.

\section{A. International example}

A review of some countries revealed the SAIDI and SAIFI performance shown in "Table I". These countries also put greater emphasis on power quality [3], [11]

\section{B. Variables affecting reliability indices}

1. Longer circuits lead to more interruptions. It is easier to provide higher reliability in urban areas; line lengths are shorter.

2. The distribution supply configuration greatly impacts reliability. Long radial lines provide the poorest service; grid networks are exceptionally reliable.

3. Higher primary voltages tend to be more unreliable, mainly because of longer lines.

Table 1. EUROPEAN RELIABILITY PERFORMANCE, WITH MAJOR EVENTS

\begin{tabular}{|c|c|c|}
\hline Country & SAIDI & SAIFI \\
\hline Austria & 72 & 0.9 \\
\hline Denmark & 24 & 0.5 \\
\hline France & 62 & 1.0 \\
\hline Germany & 23 & 0.5 \\
\hline Italy & 58 & 2.2 \\
\hline Nerthlands & 33 & 0.3 \\
\hline Spain & 104 & 2.2 \\
\hline UK & 90 & 0.8 \\
\hline
\end{tabular}

*Source: council of European energy regulator ASBL (2008) 4th benchmarking report on the quality of electricity supply.Brussel CEER.

Faults and interruptions have significant year-to-year variation because weather conditions vary significantly, age of electrical equipment, or performance of protective systems. These factor variations are translated into variations in the number of faults and in reliability indices.

\section{Relation between SAIFI \& CAIDI}

The envelope of "acceptable supply" for relation between frequency of interruptions and longest duration per interruption is shown in "Fig.3".

- Find optimum and customer satisfied: "A" area, represents the area of biggest reliability, move towards the origin, the performance will be better.

- Customer dissatisfied: Balanced area represents the lower reliability, divided to:

- Region "B" indicates for the excess number of interruptions but for short time durations.

- Region "C" indicates for little number of interruptions but for long time durations.

- Vertical axis " $X$ " and horizontal axis "Y" give the reliability indices threshold.

\section{Performance indicators for EDC}

"Table 2" and "Table 3" represent the development of SAIFI and CAIDI during 2011:2014 for EDCs. If indicators through the three years are abnormal, (as CAIDI for EDC3 \& EDC5), the stray number must be dropped out.

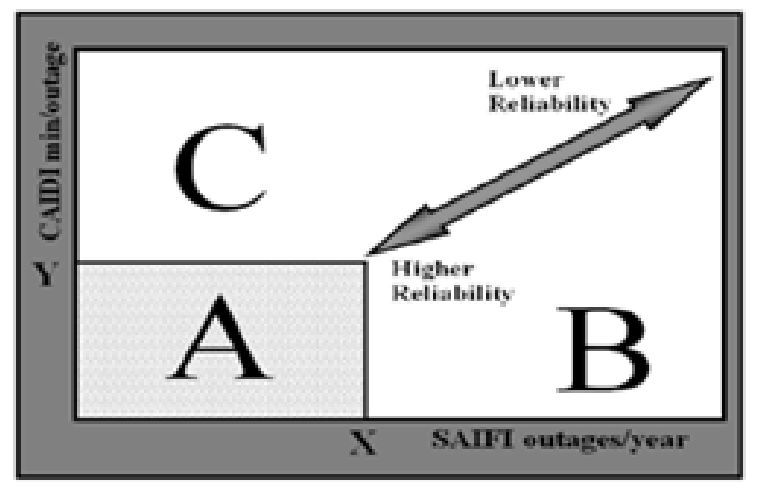

Fig.3. Relation between SAIFI and CAIDI 
Table 2. DEVELOPMENT OF CAIDI FOR EDCS

\begin{tabular}{|c|c|c|c|c|c|}
\hline \multicolumn{7}{|c|}{ CAIDI } \\
\hline Company & $\mathbf{2 0 1 1}$ & $\mathbf{2 0 1 2}$ & $\mathbf{2 0 1 3}$ & $\mathbf{2 0 1 4}$ & average \\
\hline EDC1 & 102.4 & 117.761 & 107.852 & 97.062 & 106.269 \\
\hline EDC2 & 31.768 & 27.12 & 24.118 & 21.758 & 26.191 \\
\hline EDC3 & 54.851 & 18.483 & 18.906 & 9.509 & 25.437 \\
\hline EDC4 & 143.577 & 2.094 & 7.576 & 67.048 & 55.074 \\
\hline EDC5 & 40.071 & 40.416 & 41.76 & 36.177 & 39.606 \\
\hline EDC6 & 67.841 & 48.567 & 27.377 & 28.104 & 42.972 \\
\hline EDC7 & 23.879 & 24.474 & 88.877 & 106.13 & 60.84 \\
\hline EDC8 & 152.413 & 106.931 & 103.703 & 108.7 & 117.937 \\
\hline EDC9 & 77.801 & 83.167 & 130.727 & 171.301 & 115.749 \\
\hline
\end{tabular}

Table 3. DEVELOPMENT OF SAIFI FOR EDCS

\begin{tabular}{|c|c|c|c|c|c|}
\hline \multicolumn{7}{|c|}{ SAIFI } \\
\hline Company & $\mathbf{2 0 1 1}$ & $\mathbf{2 0 1 2}$ & $\mathbf{2 0 1 3}$ & $\mathbf{2 0 1 4}$ & average \\
\hline EDC1 & 0.435 & 0.495 & 0.423 & 0.341 & 0.4235 \\
\hline EDC2 & 0.192 & 0.169 & 0.134 & 0.131 & 0.1565 \\
\hline EDC3 & 0.149 & 0.934 & 0.958 & 0.764 & 0.70125 \\
\hline EDC4 & 0.452 & 0.405 & 0.442 & 0.445 & 0.436 \\
\hline EDC5 & 0.66 & 0.533 & 1.01 & 1.009 & 0.803 \\
\hline EDC6 & 3.01 & 2.145 & 0.095 & 0.081 & 1.3328 \\
\hline EDC7 & 0.121 & 0.117 & 1.388 & 0.559 & 0.5463 \\
\hline EDC8 & 0.289 & 0.169 & 0.464 & 0.225 & 0.287 \\
\hline EDC9 & 1.59 & 2.237 & 0.221 & 0.179 & 1.0568 \\
\hline
\end{tabular}

The relation between SAIFI and CAIDI for 9 EDCs during 2011:2014 is presented in "fig. 4".

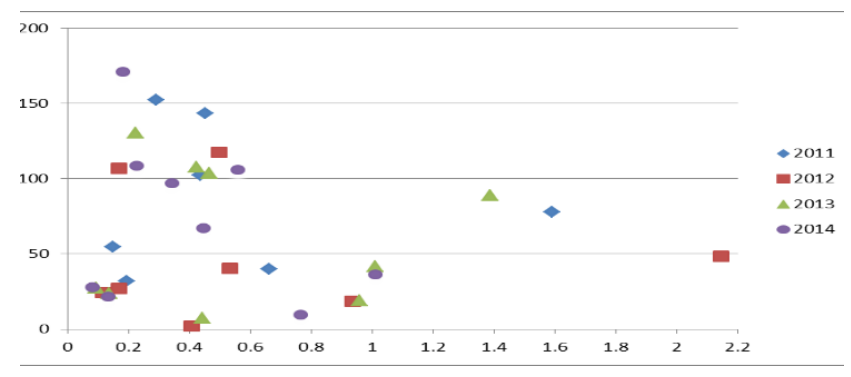

Fig.4. relation between SAIFI\&CAIDI for EDCs (data 2011-20122013-2014)

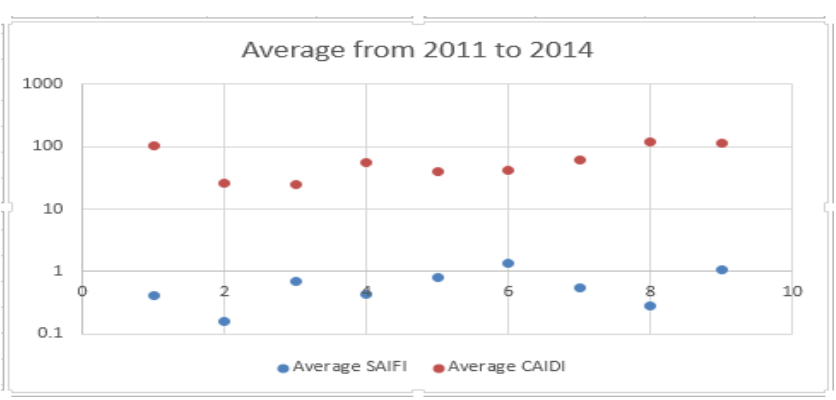

Fig.5 Relation between SAIFI and CAIDI fir EDCs in 2014

"Fig.5" represents the envelope of "acceptable supply "for relation between SAIFI and CAIDI for EDCs in 2014.

According to IEEE1366, 2001 the target of SAIFI and CAIDI are 1.0 and 90 minute respectively, they are close to the calculated threshold values

Notes:

1. SAIFI and CAIDI are improved in 2014 for most EDCs.

2. EDC2 and EDC3 are in optimum area

\section{CONCLUSION}

There is increasing demand from consumers for more reliable and economical electric power. Many factors share to evaluate the reliability of a power network: design, planning, operation and maintenance and faults; which have their contributed input to all power network reliability.

The main measures to improve reliability: adequate maintenance, adoption of preventive maintenance rather than break down maintenance, improving power quality and ensuring coordination protection settings.

The reliability indicators for electric network in Egypt are used by EEHC and Egypt Era to benchmark performance and scenariotize investments in generation, transmission and distribution network, to improve performance. Also, these indicators are used by system planners and operators as a channel to improve the level of customer service.

Reliability level for its delivery facilities and, where appropriate to improve performance.

The results are the reliability performance objectives for EDCs, ETC, and EPCs shall have threshold objective designed to help maintain the acceptable envelop.

\section{REFERENCES}

[1] L.Goel, "based reliability studies of a distribution test system," Monte Carlo simulation [Online]. Available: http://www.sciencedirect.com

[2] H.Haroonabadi, and M.R.Haghifam, "Generation 
Reliability Assessment in Power Markets Using Monte Carlo simulation and intelligent system," World Applied Sciences Journal 5(3):302-310, 2008.

[3] Dennis Hesseling en Mahir Sari, " The introduction of quality regulation of electricity distribution in the Netherlands," European Energy Law report $ш, 2006$.

[4] Kamelia Youssef and Hafez El-Salmawy,"Indices of supply: Benchmarking EDCs in Egypt," MEPCON 2009, Assuit University,Egypt.

[5] Kamelia Youssef and Hafez El-Salmawy,"Power Quality: reliability indices for transmission system in Egypt, "The conference of the power quality and harmonics (JCNC)," Amman, Jordan.

[6] Mahmud Fatuhi, Firuzabad, and Saeed Afshar, "Reliability analysis in Electrical Distribution system considering preventive maintenance applications on circuit breakers," World Academy of Science, Engineering and Technology 492009.

[7] Math BOLLEN, and Anders HOLM, "A customer oriented approach towards reliability indices," CIRED, 19th International Conference on Electricity Distribution, Vienna, 2007.
[8] Lina BERTLING, and Roland ERIKSSON, "Impact of maintenance strategy on cost and reliability of distribution systems,"CIRED 17th International Conference on Electricity Distribution, Barcelona, 2003.

[9] Michael HOLZENTHAL, Andre OSTERHOLT, "Reliability Based Planned: Reducing the Reinvestment needs of an Urban Utility," CIRED 17th International Conference on Electricity Distribution, Barcelona, 2003.

[10] Konstantin Petrov, and Daniel Grote, "Quality of supply," Presentation for ERRA Tariff Committee, April 2010

[11] "Electricity Distribution Quality of Service Report2002/2003," 14 Distribution Network Operators, Great Britain, July 2004.

[12] A. Sumper, A. Sudria, and F.Ferrer, "International Reliability Analysis in Distribution Networks," CITCEA, Universidad Politecnica de Catalunya, Spain. 